\title{
Observation of internal $x$-ray wave fields during Bragg diffraction with an application to impurity lattice location
}

\section{Citation}

Golovchenko, Jene A., Boris W. Batterman, and Walter L. Brown. 1974. Observation of Internal $\mathrm{x}$-Ray Wave Fields During Bragg Diffraction with an Application to Impurity Lattice Location. Physical Review B 10, no. 10: 4239-4243. doi:10.1103/physrevb.10.4239.

\section{Published Version}

doi:10.1103/PhysRevB.10.4239

\section{Permanent link}

http://nrs.harvard.edu/urn-3:HUL.InstRepos:29407070

\section{Terms of Use}

This article was downloaded from Harvard University's DASH repository, and is made available under the terms and conditions applicable to Other Posted Material, as set forth at http:// nrs.harvard.edu/urn-3:HUL.InstRepos:dash.current.terms-of-use\#LAA

\section{Share Your Story}

The Harvard community has made this article openly available.

Please share how this access benefits you. Submit a story.

\section{Accessibility}




\title{
Observation of internal $x$-ray wave fields during Bragg diffraction with an application to impurity lattice location*
}

\author{
Jene A. Golovchenko ${ }^{+}$ \\ Bell Laboratories, Murray Hill, New Jersey 07974 \\ and Brookhaven National Laboratory, Upton, New York 11973 \\ Boris W. Batterman \\ Cornell University, Ithaca, New York 14850
}

Walter L. Brown

Bell Laboratories, Murray Hill. New Jersey 07974

(Received 25 June 1974)

\begin{abstract}
By a simple extension of a previous experimental technique we demonstrate how the detailed standing-wave features of the internal $x$-ray wave field during the diffraction process may be observed even when a strong reflection occurs. The preliminary results reported here indicate that dynamical diffraction may be developed as a useful lattice-location technique in nearly perfect crystals.
\end{abstract}

\section{INTRODUCTION}

The dynamical theory of $\mathrm{x}$-ray diffraction predicts ${ }^{1}$ that when $x$-rays are diffracted by large single crystals, wave fields inside the crystal that contain nodal an antinodal surfaces are excited. \{The term "nodal" has been used in a loose sense. Here and in the following when refer ring to a nodal surface we generally mean a relative minimum surface of time-averaged $x$-ray intensity. For incident $x-$ rays whose electric vector lies perpendicular to the plane of incidence (i.e., $\sigma$ polarization) a perfect nodal surface of zero intensity can be achieved only when $\operatorname{Im}\left(F_{0}\right)=\operatorname{Im}\left(F_{H}\right)\left[\mathrm{F}_{H}\right.$ is the structure factor for $(h k l)$ reflection]. For the electric vector polarized in the plane of incidence ( $\pi$ polarization), genmetrical considerations alone preclude the existence of perfect nodes. Notwithstanding these results we note that a strong minimum in the standing-wave fields is achieved in both cases, as is evident from Fig. 5(b). We refer to these strong minima as nodes. $\}$ The excitation of an external diffracted beam associated with a reciprocal-lattice point $(h, k, l)$ results in internal $\mathrm{x}$-ray fields whose nodal and antinodal surfaces are parallel to and have the spacing of the $(h k l)$ planes. As the angle between the external beam and the crystal planes is varied in the vicinity of the Bragg condition, the phase relationship between the wave field surfaces and the crystal planes is also varied. The nodal surfaces move from being on the atomic planes to being between them. Such considerations apply to both the Bragg and Laue geometries. The Bragg case is somewhat more straightforward to analyze since only one wave field of the aforementioned type is excited (for each type of incident $\mathrm{x}$-ray polarization). In this paper we discuss a direct observation of the motion of the nodal surfaces.

The problem of locating the atomic positions of impurity atoms in crystals has occupied many experimentalists during the past decade. In particular, the various interactions of heavy fast charged particles with the impurities, combined with the channeling phenomena in the host crystal, has provided spatial information about the impurity ${ }^{2}$ in a rather direct way. In what follows we will discuss how the motion of the nodal surfaces of $x$-rays in a solid under conditions of strong diffraction may be used in an analogous manner.

Batterman $^{3}$ (1964) observed the angular dependence of fluorescence scattering of Mo $K \alpha$ x-rays on germanium while scanning through the Bragg condition for the (220) planes, demonstrating in a graphic way the spatial properties of the internal wave field. Then Batterman ${ }^{4}$ (1969) showed how observation of fluorescent radiation from impurities under the same condition can yield information on the impurity location. The case studied was silicon grown with $\sim 5 \times 10^{19}-\mathrm{cm}^{-3}$ arsenic concentration.

A limitation encountered in the above work was that in the angular region where one has most control over the motion of the $\mathrm{x}$-ray wave field (i.e., within the Darwin-Prins curve) the angular yield of the arsenic fluorescence was dominated by primary extinction (the depletion of $\mathrm{x}$-ray intensity with depth into the crystal due to strong coherent scattering by the atoms of the solid). Thus the big dips in Batterman's angular fluorescence yield result from primary extinction diminishing the number of arsenic atoms which the incident $\mathrm{x}$-rays can penetrate and cuase to fluoresce. (The arsenic atoms were distributed uniformly in depth throughout the sample.) Consequently, this feature is independent of the atomic location of the impurity, and indeed this strong reduction in yield served to mask the interesting effects due to motion of the internal wave fields during Bragg reflection. It of course also 


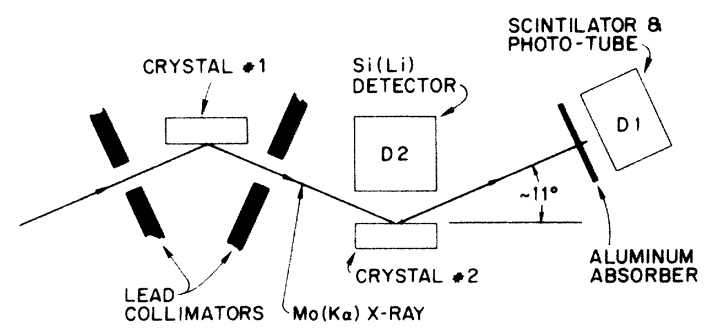

FIG. 1. Schematic illustration of experimental setup.

limits the sensitivity with which the lattice location of impurities can be determined.

In this work we report on a similar experiment, the main difference being that the arsenic atoms are contained within a distance of the surface that is smaller than the extinction length for the probing $x$-rays. Consequently, the variation in angular yield will virtually all be due to wave-field nodal and antinodal motion, extinction effects being eliminated.

\section{EXPERIMENTAL TECHNIQUE}

The experimental apparatus is illustrated schematically in Fig. 1. 17.5-keV Mo $K \alpha$ x-rays were diffracted off crystal 1 , which served as a monochrometer. The emergent $x$-rays were then directed to crystal 2 , the sample to be studied. In this case both crystals were cut with the (220) planes parallel to the surface. Detector $D_{1}$, a scintillation crystal photomultiplier tube arrangement, mon-

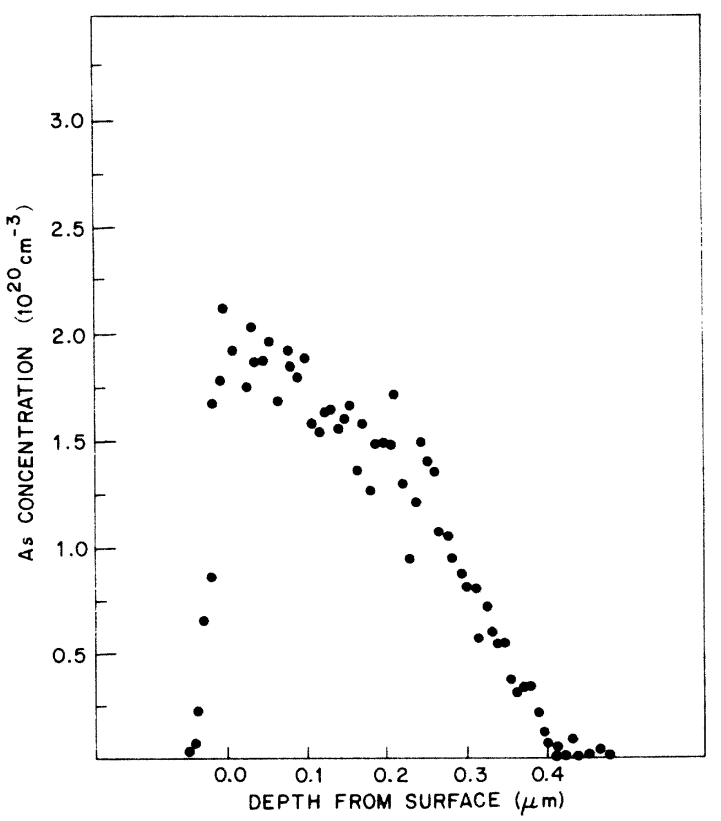

FIG. 2. Experimental backscattering results displayed as arsenic concentration in crystal 2 as a function of depth from the surface.

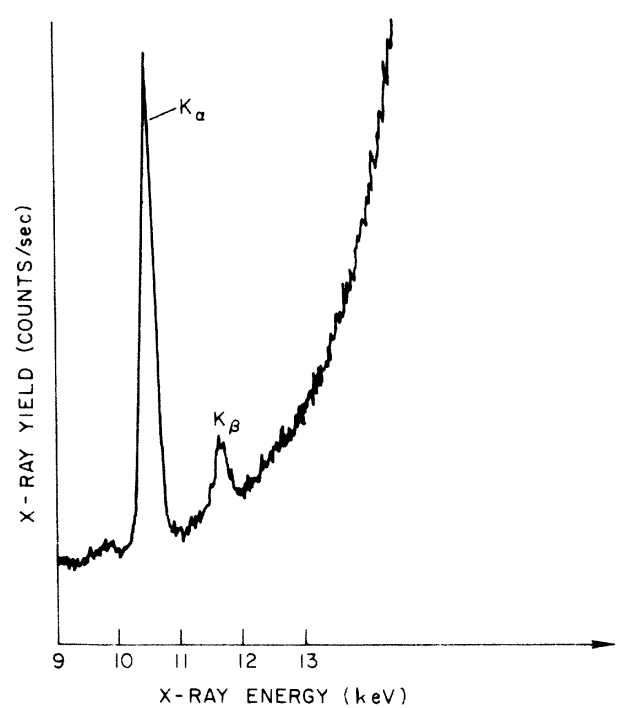

FIG. 3. Typical As-fluorescence x-ray energy spectrum from detector $D_{2}$. The background is a vestige of the thermal and Compton scattering peaks at $\sim 17 \mathrm{keV}$.

itored the diffracted beam intensity. An absorber was often used in front of this detector so that pulse-counting methods could be used without overloading the electronics. A lithium drifted silicon $\mathrm{x}$-ray detector $D_{2}$ was situated as close to the sample as possible without intercepting the incident or diffracted external beams.

The low-dislocation-density silicon sample used in this experiment was arsenic doped by diffusion at $1100^{\circ} \mathrm{C}$ for $20 \mathrm{~min}$. The depth profile of arsenic concentration was measured by Rutherford backscattering ${ }^{1}$ with 1.9-MeV helium ions, and the results are shown in Fig. 2. The surface concentration is $2 \times 10^{20} \mathrm{~cm}^{-3}$ and the depth distribution is not a typical complementary error function. Such results are common for high-dose shallow diffusions of arsenic. ${ }^{5}$ Notice should be taken that the backscattering experiment was performed after the $\mathrm{x}-$ ray measurements because it is known that relatively low helium-ion fluxes cause arsenic atoms to move off lattice sites. ${ }^{6}$

The Si ( $\mathrm{Li}$ ) detector resolution was about $200-\mathrm{eV}$ full width at half-maximum, and a typical spectrum containing the arsenic fluorescence is shown in Fig. 3. The arsenic fluorescence peaks are riding upon a background due to detector tailing from higher-energy Compton and thermal scattering of the incident Mo $K \alpha$ x-rays. This background was dependent on the crystal orientation near the Bragg condition and was subtracted to give the true arsenic yield in the experiment.

The data points in Fig. 4(a) show the angular yield in detector $D_{1}$ of $x$-rays diffracted from the silicon (220) planes. In Fig. 4(b) the corresponding angular yield of As $K \boldsymbol{\alpha}$ fluorescent radiation as 
seen by $D_{2}$ is shown. Each point represents 500 sec of counting, which yields about $10^{3}$ counts. The $\mathrm{x}$-ray tube conditions were $50 \mathrm{kV}$ anode potential at $32 \mathrm{~mA}$. The random yield was determined by tilting the sample $\sim 1^{\prime}$ ' of arc from the Bragg condition.

\section{DISCUSSION AND ANALYSIS}

In the following we shall discuss, without going into great detail, how the experimental results can be accounted for within the dynamical theory of $x-$
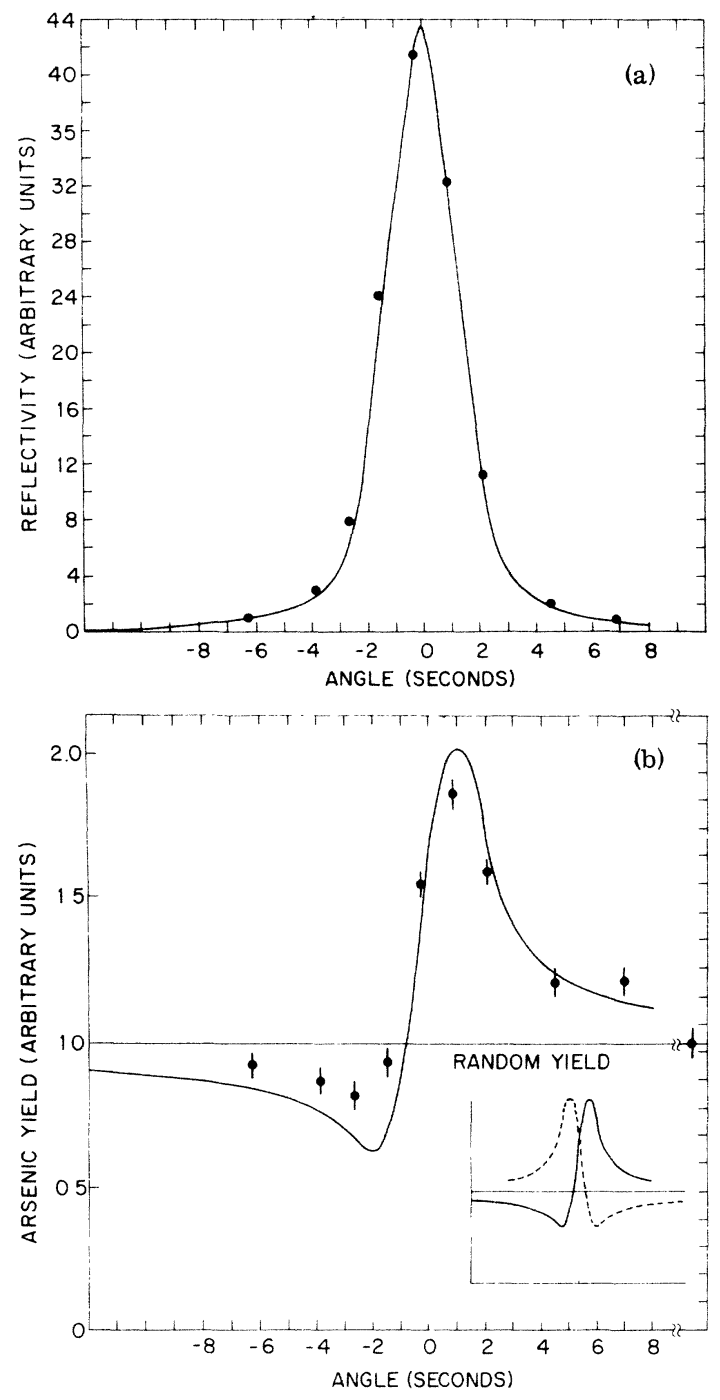

FIG. 4. (a) Closed circles indicate angular yield in detector $D_{1}$ as crystal 2 is scanned through the Bragg condition. Solid curve is theoretical calculation described in text. (b) Data points are experimental As $K_{\alpha}$-fluorescence $x$-ray yields in detector $D_{2}$ as crystal 2 is scanned through the Bragg condition. Solid curve is theoretical calculation described in the text. The sketch in the inset contrasts the different results expected for impurity directly between planes (dotted line) and impurity in the planes (solid line).
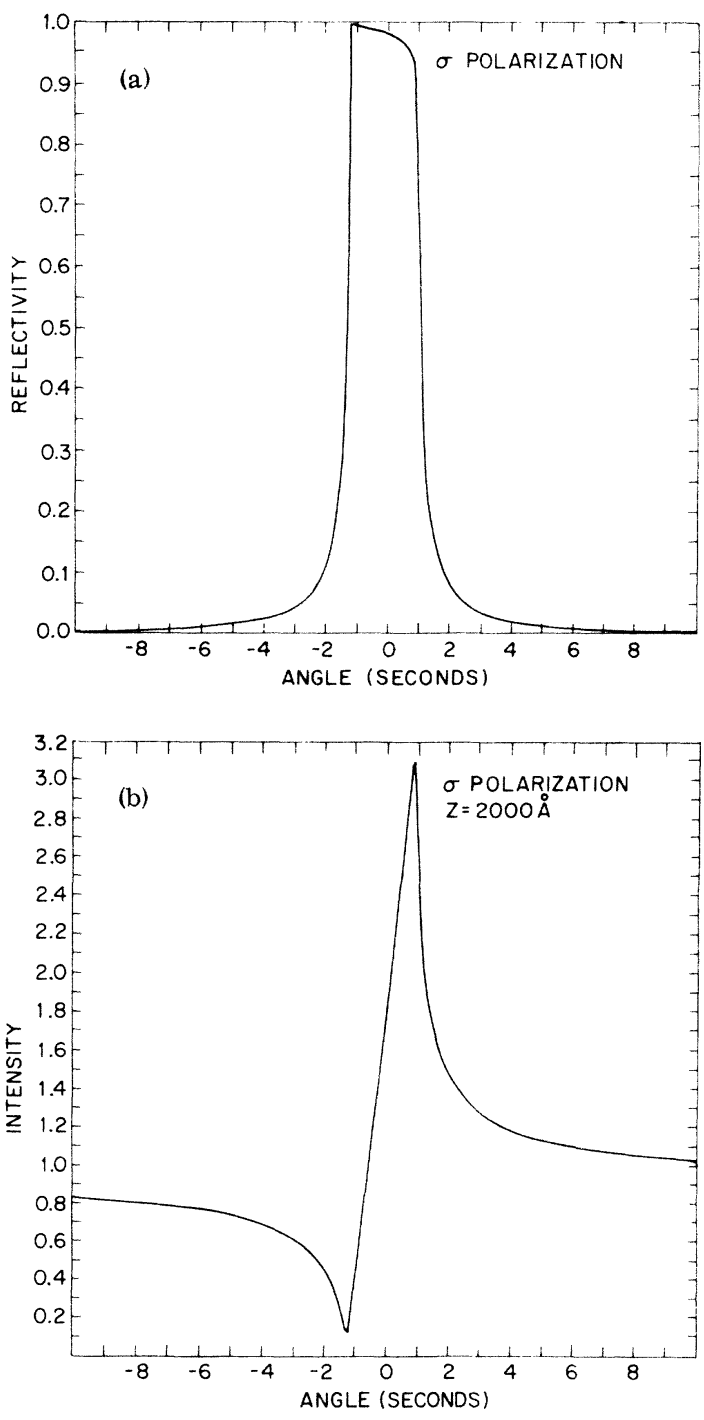

FIG. 5. (a) Calculated reflectivity from Eq. (2) of text for the $\sigma$ polarization state. The result for the $\pi$ state is essentially similar. (b) $x$-ray intensity on the (220) planes vs angle of incident $\sigma$ polarized x-ray beam. The result for $\pi$ polarization is essentially similar.

ray diffraction. For the derivation of formulas used in this section the reader is referred to Refs. 2 and 3.

$X$ rays incident upon a crystal lattice will diffract strongly from the $(h k l)$ planes if they satisfy the Bragg condition

$$
2 d(h k l) \sin \theta=\lambda,
$$

where $d(h k l)$ is the spacing between $(h k l)$ planes, $\lambda$ is the $\mathrm{x}$-ray wavelength, and $\theta$ is the angle between the planes and the direction of the incident beam. Actually there is a range of angles, near the Bragg condition (1), within which a strong diffracted beam will be excited. We restrict ourselves now to the 
TABLE I. Constants used in the calculation.

$$
\begin{aligned}
F_{0} & =112.0+i 0.688 \\
F_{H}(220) & =70.0+i 0.661 \\
\lambda\left(M_{0} K_{\alpha}\right) & =0.711 \AA \\
\mu_{0} & =17.1 \mathrm{~cm}^{-1}
\end{aligned}
$$

geometry of this experiment, i.e., a symmetric Bragg reflection. The theoretical description of the reflectivity $R^{2}$ from the surface of an infinitely thick perfect crystal is contained in the DarwinPrins curve analytically given by

$$
R^{2}=\left|\eta \pm\left(\eta^{2}-1\right)^{1 / 2}\right|^{2}
$$

with the complex quantity $\eta$ being

$$
\eta=\frac{-\Delta \theta \sin 2 \theta+\Gamma F_{0}}{\Gamma|P|\left(F_{H} \bar{F}_{H}\right)^{1 / 2}} \text {. }
$$

Here $\Delta \theta$ is the deviation from the Bragg condition (1), $F_{H}$ and $\bar{F}_{H}$ are the structure factors for the reflections $(h k k)$ and $(\bar{h} \bar{k} \bar{l})$, respectively, $\Gamma=$ $=\left(e^{2} / m c^{2}\right) \lambda^{2} N / \pi$ with $N$ the number of unit cells per unit volume, and $P$ is 1 or $\cos 2 \theta$ for the $\sigma$ and $\pi$ polarization states. A plot of $R^{2}$ vs $\Delta \theta$ is shown in Fig. 5(a).

In order to compare the data in Fig. 4(a) with this result, it is essential to realize that for our experimental arrangement the incident $x$-ray beam on crystal 2 has an angular width governed by the reflectivity curve from crystal 1 , the monochrometer. Thus the total reflected intensity in detector $D_{1}$ should be a convolution of Eq. (2) with itself. This is the solid curve in Fig. 4(a), taking account of both polarizations. The vertical scale is normalized to the data and the horizontal zero is chosen to center the data points around the calculated curve. The shape of the experimental result is in quite good agreement with the calculation as it should be if both crystals are of high quality.

The expected variation of arsenic yield with angle may be calculated in a simple way based on the assumption that all the arsenic atoms are lying in the (220) planes. (Arsenic is known to be highly substitutional from previous measurements. ${ }^{8}$ ) For this case the yield of arsenic fluorescence at any given depth can be taken to be proportional to the silicon absorption and the local arsenic concentration. Using an energy conservation argument and the dynamical theory prediction for the imaginary part of the internal $x$-ray field wave vector, one finds that in a depth increment $d z$ the fraction of incident energy absorbed, $d I$, is

$$
d I=\left(1-R^{2}\right) \mu(\eta) e^{-\mu(\eta) \varepsilon},
$$

with

$$
\mu(\eta)=\frac{\mu_{0}}{\sin \theta}\left[1-|P| \operatorname{Im}\left(\frac{F_{H}}{\operatorname{Im}\left(F_{0}\right)}\left[\eta \pm\left(\eta^{2}-1\right)^{1 / 2}\right]\right)\right] .
$$

The sign in Eqs. (2) and (5) is chosen to make $R^{2}$ $\leq 1$. Here $\mu_{0}$ is the linear absorption coefficient. For illustration Eq. (4) is also plotted in Fig. 5(b) for a depth of $0.2 \mu \mathrm{m}$ into the surface for the $\sigma$ polarization state. The dip on the low-angle side of of the Bragg condition is characteristic of the nodes of the x-ray field lying on the (220) planes while the peak on the high-angle side shows the antinode lying on the planes. In order to compare with the data, Eq. (4) multiplied by a linear approximation to the impurity depth distribution is integrated over the depth $z$ and convoluted with the reflectivity curve from crystal 1. The result of this exercise (including both polarization states) is the solid curve in Fig. 4(b). Table I contains values of the parameters used in the calculation.

In Fig. 4(b) the vertical scale of the measured points has been normalized to the calculated curve at the random value. We feel at this preliminary stage that the agreement between theory and results is satisfactory, although it is hoped that the disparity that does exist in the angular arsenic yield may be accounted for in the future. Possible explanations for this disagreement are crystal strain and arsenic atoms not sitting exactly at substitutional position. It is $\mathrm{known}^{5}$ that at high concentrations of arsenic in silicon a significant fraction of the arsenic is electrically inactive, which suggests that arsenic complexes as well as substitutional $\mathrm{As}^{+}$ions may exist in the crystal. The lattice strain and exact positions of the atoms in the proposed complexes are not known. These considerations could easily affect our results.

In order to give the reader an intuitive feeling for the kind of sensitivity one has to the position of As in the silicon lattice, we note from symmetry that if the arsenic atoms were situated directly between the planes, the data would be relfected around the angle corresponding to the maximum yield in the convoluted Bragg reflection. The sketch in the inset of Fig. 4(b) illustrates this result. Thus the large increase in arsenic fluorescent yield would be on the low-angle side and the dip on the highangle side.

\section{ACKNOWLEDGMENTS}

The authors would like to express their gratitude to R. B. Fair and W. F. Flood for preparing the sample used in this work. J. B. Roberto provided valuable assistance in setting up and in performing the experiment. Marilyn Robbins's help in generating the calculated curves presented above is also greatly appreciated. Finally we acknowledge useful discussions with Leonard Feldman, and one of us (J. G.) would like to thank A. N. Goland for encouragement during the course of this work. 
*Research carried out in part under the auspices of the U.S. Atomic Energy Commission.

$\dagger$ Present address: Institute of Physics, Aarhus University DK 8000, Aarhus C, Denmark.

${ }^{1} \mathrm{R}$. W. James, The Optical Principal of the Diffraction of $X$-Rays (Bell, London, 1950); B. W. Batterman and H. Cole, Rev. Mod. Phys. 36, 681 (1964).

${ }^{2}$ J. W. Mayer, L. Eriksson and J. A. Davies, Ion Im- plantation in Semiconductors (Academic, New York, 1970).

${ }^{3}$ B. W. Batterman, Phys. Rev. 133, A759 (1964).

${ }^{4}$ B. W. Batterman, Phys. Rev. Lett. 22, 703 (1969).

${ }^{5}$ R. B. Fair and G. R. Weber, J. Appl. Phys. 44,273 (1973).

${ }^{6}$ E. Dimini, J. Haskell, and J. W. Mayer, Appl. Phys. Lett. 20,237 (1972). 